\title{
Author Correction: Quantum metasurfaces with atom arrays
}

R. Bekenstein (1), I. Pikovski, H. Pichler, E. Shahmoon, S. F. Yelin and M. D. Lukin (D)

Correction to: Nature Physics https://doi.org/10.1038/s41567-020-0845-5, published online 30 March 2020.

In the version of this Article originally published online, a present address was missing for author E. Shahmoon: Department of Chemical \& Biological Physics, Weizmann Institute of Science, Rehovot, Israel; this has now been added. All versions of the Article have been amended.

Published online: 22 April 2020

https://doi.org/10.1038/s41567-020-0912-y

๑ The Author(s), under exclusive licence to Springer Nature Limited 2020 